\title{
Chris Rauseo
}

Universität Valenciennes

DOI: $10.19195 / 0435-5865.141 .3$

\section{Vaterländische Unwahrheiten. Fontane und Kleist}

Fontane hat lange gewartet, bis er anfing, Kleist zu lesen. Allem Anschein nach kannte er vor 1872 nichts von ihm - was bei einem preußischen „homme de lettres" von über 50 Jahren doch etwas überrascht, handelte es sich doch, wie man rückblickend feststellen kann, um den bedeutendsten Vorgänger Fontanes in der dichterischen Gestaltung preußischen Wesens. Warum er Kleist so lang aus dem Weg ging, ist unklar, wie meistens in solchen Fällen. Ahnte oder fürchtete er eine zu große Nähe? Spürte er, dass Kleist bei Fragen, die ihn auch beschäftigten, zu sehr andere Wege gehen musste? Konnte es sogar sein, dass er sich mit 30 oder 40 oder 50 sagte: „Das ist nichts für dich“? Dies alles liegt im Bereich der reinen Spekulation, Belege sind nicht vorhanden. Seitens der Gattungen zumindest hatte Fontane keinen Grund zur Scheu. Kleist schrieb Dramen und Erzählungen, sowie hoch konzentrierte Anekdoten, also fernab von Fontanes bevorzugten Gebieten. Als dieser jedenfalls seine Kleist-Abstinenz beendete, ging er gründlich ans Werk und verfasste ausführliche, private Aufzeichnungen über drei Dramen und fünf Erzählungen. Unter den letzteren bespricht er - neben dem Bettelweib von Locarno, der Verlobung in St. Domingo, der Marquise von O... und Michael Kohlhaas - durchaus irritiert Die heilige Cäcilie oder die Gewalt der Musik. Dass protestantische Bilderstürmer von der Reinheit, der Unschuld und dem Wunderglauben katholischer Nonnen moralisch, geistig und seelisch entwaffnet würden, möge noch hingehen - doch nur dann, wenn die Geschichte von einem Katholiken erzählt worden wäre. Was Kleist bekanntlich nicht war: und „so macht das ganze den Eindruck einer Verherrlichung des Katholizismus, zu dem sich ein Protestant nicht hergeben darf.“1 Auch Dichter haben loyal zu sein, sie dürfen mit gegnerischen Partien auch nicht im Namen der Kunst fraternisieren: „Ich darf mich aber nicht hinsetzen und eine Geschichte schreiben, in der Napoleon I. recht und die

${ }^{1}$ Theodor Fontane, Ach, es ist schlimm mit den Dichtern. Über Literatur, Autoren und das Publikum, Berlin 1999, S. 103. 
Königin Luise unrecht hat.“2 Dies sei einem französischen Kollegen gern gestattet: „Ich aber darf es nicht.“3 Die beinahe obsessive Wiederholung des Verbs „dürfen“, verbunden mit der Verwendung des Pronomens ,ich“, verwandelt Fontanes Behauptungen bisweilen in Mahnungen an sich selbst: so wie Kleist verfährst du nicht, selbst wenn du es wolltest. Für einen Preußen ist Kleist hier kein Vorbild: „So durfte Kleist nichts schreiben, was das Klostertum im Glanz und die Bewegung gegen dasselbe als einen frechen Unfug darstellt. Die Kunst hat nicht das Recht, sich vom Leben zu lösen und Glauben und Vaterland zu ignorieren, sobald es sich um einen guten Stoff handelt. So viel ist auch der beste Stoff nicht wert."4

An das hier postulierte Gebot der Treue schließt sich die Warnung vor der Selbstverleugnung an. Wer gegen das eigene Land schreibt, hat nicht nur keinen Ort, sondern auch kein Ich mehr. So weit ging Kleist allerdings nicht, da die $\mathrm{Hei}$ lige Cäcilie in dieser Hinsicht, und nach Fontanes Kriterien, ein Einzelfall blieb. Dennoch hat sich Kleist, aus Fontanes Sicht, in einem Drama, das er selbst ,vaterländisch" nannte, eines weiteren Vergehens gegen das Land seiner Väter schuldig gemacht. ${ }^{5}$ Zwar handelt es sich nicht um einen Fall von Illoyalität wie in der Reformations-Erzählung. Aber doch noch um Untreue, wenn auch in anderer Gestalt. Im Schauspiel Prinz Friedrich von Homburg erblickt Fontane überall Verrat an der preußischen Geschichte, obwohl die preußische Sache letztendlich siegreich behauptet wird. Die Geschichtsverfälschung berührt sowohl die Ereignisse um die Schlacht von Fehrbellin (1675) als auch die Person und den Charakter des Helden. Von Ignoranz der Tatsachen seitens des Verfassers konnte keine Rede sein, war doch dieser „ein pommersch-brandenburgischer Edelmann, ein Kleist, ein gewesener Gardeoffizier [...], der diese Verstöße macht und im Kleinen und Großen alles so unbrandenburgisch darstellt wie nur möglich." ${ }^{66}$ Insbesondere das systematische Umformen aller Sitten und Gesetze des preußischen Militärwesens (der zum Tode verurteilte Prinz darf selber entscheiden, ob die Strafe vollzogen wird oder nicht) ruft Fontanes Verwunderung hervor: „Diese Art von Gemütlichkeit in Fragen, wo bei den Hohenzollern immer die Gemütlichkeit aufhörte, nämlich in Militär- und Disziplinfragen, ist durchaus unhistorisch, unbrandenburgisch und gibt ein ganz falsches Bild von Zeit, Land und Menschen."7

Dabei ist das Unhistorische nicht einmal das Bedenklichste an diesem vaterländischen Drama, in welchem das Vaterland so verfremdet und befremdend

2 Ebd., S. 104.

${ }^{3}$ Ebd.

${ }^{4}$ Ebd.

5 Brief von Kleist an den Verleger Reimer vom 21. Juni 1811: „Wollen Sie ein Drama von mir drücken, ein vaterländisches (mit mancherlei Beziehungen) namens der Prinz von Homburg, das ich jetzt eben anfange, abzuschreiben?“ (Kleist, Briefe, Frankfurt am Main 1986, S. 477).

${ }^{6}$ Fontane, op. cit., S. 95. Die Überschrift dieser Aufzeichnung trägt den Titel „Der Prinz von Hessen-Homburg“, wodurch Fontane darauf aufmerksam macht, dass Kleist sogar den Namen seines Helden ungetreu wiedergibt.

${ }^{7}$ Ebd., S. 96. 
wirkt. Denn der vermeintliche Held ist gar keiner; höchstens am Schluss, aber dann, aufgrund des bereits Vorgefallenen, ist der Glaube an ihn längst verspielt. Er gehört laut Fontane zu denen, die „eitle, krankhafte, prätentiöse Waschlappen, aber keine Helden“ sind - „Kerle, die [...] immer nur Unheil gestiftet haben und die immer nur in kranker Zeit oder von kranken Gemütern gefeiert worden sind. “8 Deren Verwerflichkeit verbietet sogar, dass ein Dichter sich mit ihnen beschäftigt: „Die Schaustellung solcher Kleinheit und Misere, in Gestalten, die erheben sollen, gehört nicht in die Kunst. "'9 Einige Aspekte des Dramas, vor allem des Prinzen Überwindung der ihn erniedrigenden Todesangst, wären durchaus wirkungsvoll, wenn ,der ganze Stoff dem Historischen entrückt ${ }^{* 10}$ und in märchenhafter Unbestimmtheit angesiedelt worden wäre. Und selbst die Bereitschaft, Kleist gewisse bewusste Anachronismen zuzugestehen (etwa: den Prinzen Friedrich für einen Vorläufer des Prinzen Louis Ferdinand - Kleists Zeitgenossen - zu halten) scheitert daran, dass letzterer bei aller Exaltiertheit das Militärische so ernst nahm, wie es sich für einen preußischen Prinzen seit jeher gehört. ${ }^{11}$

Auffallend ist natürlich Fontanes Strenge beim Abzirkeln des Zulässigen und Statthaften in vaterländischer Dichtung. Vielleicht lag es daran, dass er zur selben Zeit (1872) gerade an dem letzten seiner insgesamt drei Bücher über Bismarcks drei erfolgreiche Kriege arbeitete - da war historische Treue selbstverständlich Pflicht. Vielleicht hing die Strenge aber noch mehr mit der noch nicht abgeschlossenen Arbeit an seinem ersten Roman Vor dem Sturm zusammen, den er wiederholt als ,vaterländisch“ 12 bezeichnete und der sich keine so massiven Abweichungen von der Historie gestattet wie Kleists Drama. Allerdings erweist es sich stets als ein heikles Unterfangen, Fontanes Meinungen überhaupt, und speziell seine vermeintlichen Glaubenssätze in ästhetischen Fragen, mit Hilfe der Biographie, der Chronologie oder einer wie immer auch gearteten „Entwicklung“ zu begründen. Fontanes ästhetische Positionen bzw. Haltungen sind in hohem Maße von Empfindungen abhängig, die sich im Theater, im Museum oder beim Lesen seiner bemächtigen. Die Kunstregeln, die er gelegentlich (wie hier bei Kleists Homburg) daraus ableitet, wirken meist fest, sind aber selten so stark, dass ein entgegen gesetzter Eindruck sie nicht abschwächen oder gar ganz außer Kraft setzen kann. In den selben Aufzeichnungen, in denen Kleists Homburg so unerbittlich verdammt wird, berichtet Fontane von seiner Bewunderung für die Novelle Michael Kohlhaas. Weil er zumindest deren erste Hälfte als ,,vollendet“ (nicht zuletzt wegen der „historische[n] Bedeutendheit des Inhalts") betrachtet, fällt das Unwahre in der

${ }^{8}$ Ebd., S. 96.

${ }^{9}$ Ebd., S. 97.

10 Ebd., S. 98.

11 Ebd., S. 99.

${ }^{12}$ Vgl. den Abschnitt „Zeit- und Sittenbild“. „Vaterländischer Roman“ im Kommentar von Christine Hehle in: Theodor Fontane, Vor dem Sturm, Band I/II, Berlin 2011, S. 380-382. 
Darstellung des geschichtlichen Kontexts kaum ins Gewicht. ${ }^{13}$ Der Tadel des ersten Herausgebers Tieck berührt ihn nicht übermäßig: „Im ,Kohlhaas’ ist Kostüm, Szenerie, Lokalität alles falsch (Tieck führt dies ausführlich aus), aber Kohlhaas ist persönlich-richtig als Kohlhaas und ist zeitbildlich-richtig als märkischer Roßkamm und schroffer Rechtscharakter des 16. Jahrhunderts geschildert." ${ }^{14}$ Wenn man also wählen muss: das Märkische, Brandenburgische und bald darauf PreuBische ist nicht in der Exaktheit der Schilderungen von Konkretem zu suchen, sondern in der Stimmigkeit der Abstraktionen, die von der Handlung vermittelt werden. Bei Kohlhaas lautet die Abstraktion Rechtsempfinden; da dieses märkisch ist, wirkt es in seiner Verkörperung schroff. Die fehlende Konkretheit in der „Lokalität" wird wettgemacht durch die Konsequenz, mit der die spezifisch „lokale“ Verwirklichung des Rechtsgefühls stattfindet.

Das Problem der geschichtlichen Treue in der Kunst begleitet Fontane sein Leben lang, was unter anderem eine Folge der ersten von ihm praktizierten Gattung sein dürfte - der Ballade, in der ja ein Mensch oder eine Tat nicht so sehr beschrieben, als vielmehr besungen wird und wo alles auf einen Punkt hinausläuft. Vereinfachungen der historischen Wirklichkeit kommen darin natürlich vor. Fontane versucht, wenn er kann, abzuwägen. Der Historismus, der Fontanes Zeitalter so prägt, hat aber auch bei ihm Spuren hinterlassen. Goethes Egmont begegnet er 1870 nur noch mit Hohn: „Diese Egmont-Gestalt, das Entzücken unserer Jugend, ist uns heute einfach ein Greuel, eine historische Sünde. Wer fünfzig Jahre alt ist, Geschichte gelesen und in sich aufgenommen hat, kann dem ,Heros deutscher Nation' dieses Attentat gegen eins der schönsten Kapitel der Geschichte der Menschheit nicht verzeihen." 15

Fontanes Sinn für die Zeitgebundenheit gerade - wie in Egmont - dramatischer Handlungen führt sogar dazu, dass der Kritiker an der Aufführbarkeit von Molières Tartuffe ${ }^{16}$ oder gar der urpreußischen Minna von Barnhelm Lessings ${ }^{17}$ zweifelt: so groß sind mittlerweile die Unterschiede zur Gesellschaft des späten 19. Jahrhunderts. In der Tartuffe-Rezension von 1885 heißt es von Molières Komödien:

Es sind jetzt Sittenbilder aus einer anderen Zeit, Sittenbilder, die wir nicht bloß zu respektieren, sondern um ihrer hohen Kunst willen auch zu bewundern haben, aber wir stehen ihnen fremd gegenüber. Was man schon bei der Lessingschen „Minna von Barnhelm“ störend empfindet: daß sich alles verändert hat und solche Menschen und Verhältnisse nicht mehr existieren, das empfindet man verdreifacht den Molièreschen Stücken gegenüber. ${ }^{18}$

\footnotetext{
${ }^{13}$ Fontane, Dichtern (vgl. Fußnote 1), S. 106.

14 Ebd., S. 107-108.

15 Theodor Fontane, Die Saison hat glänzend begonnen. Theaterkritiken, Berlin 1998, S. 66.

16 Ebd., S. 54-57.

17 Ebd., S. 57-59.

18 Ebd., S. 56. In der Minna-Rezension von 1870 erscheint Lessings Komödie beinahe wie ein altpreußisches Familienbuch, in das man gerührt, aber auch ein wenig verwundert schaut: ,nur eine gewisse Vollendung der Darstellung, die uns das Ganze dann wie eine Reihe lebender Bilder, die
} 
Dem Dichter lauert also überall Gefahr. Greift er historische Stoffe auf, droht ihm der Verrat am Stoff, wenn er diesen mutwillig umformt; wählt er dagegen zeitgenössische Themen, läuft er das Risiko der Kurzlebigkeit, wenn die Handlung mit ihrer Zeit in zu exklusiver Vertrautheit steht. Der von Fontane eigentlich bewunderte Gottfried Keller sucht einen Ausweg aus diesem Dilemma, indem er - etwa in den Leuten aus Seldwyla - „Historie, Kultur- und Sittengeschichte“ seinem Märchenton und einem ,poetischen Einfall“ unterordnet. ${ }^{19}$ Kellers Vermengung von Märchen und Historie mag das Publikum entzücken, löst aber bei Fontane Unbehagen aus:

Die meisten Leser, unbefangen wie Kinder, weil sie mit ihrem historischen Wissen auf einem Kinderstandpunkt stehen, gehen leicht darüber hin, bemerken es nicht oder bestreiten wohl gar die Berechtigung dieser Ausstellung; wer aber die begangenen Fehler als solche erkennt, zuckt mitunter zusammen und wird unwirsch, weil ihn die Unwahrheit der mit einer gewissen Prätension auftretenden Situationen verdrießt. ${ }^{20}$

Eine bessere Lösung zur Überwindung der gegensätzlichen Ansprüche von Historie und Fantasie kommt aus Großbritannien. Für den Roman ist sie schottischer Herkunft und stammt von Walter Scott, wie Fontane ein Romancier, der zuerst Balladendichter war. In seinem Erzählwerk gelingt Scott die Quadratur des Kreises. Er vermittelt die „Geschichte seines Landes“ und zugleich die „scharf gezeichneten Lokalitäten, die den Hintergrund oder die Bühne für all dies Geschehene bildeten“ - und trotz aller Exaktheit auch noch, ,,als wichtigstes, den in seiner Simplizität so tief treffenden Klang der alten Balladen." ${ }^{21}$ Dadurch wurden seine Romane, die so sehr in Scotts auf den ersten Blick so entlegener Heimat wurzeln, bei aller scheinbaren Provinzialität universal:

Erst Walter Scott war es, der uns die Geschichte, die landschaftlichen Schönheiten, die Sagen und Sitten Schottlands bis zu einem Grade erschloß, daß man behaupten darf, die Schicksale der Stuartpretendenten von 1715 und 1745 zählen zu den bekanntesten und beliebtesten Kapiteln, die die Weltgeschichte aufzuweisen hat. Das liegt nicht in dem Gewicht der Dinge selbst, das liegt lediglich in der poetischen Bedeutung, die ihnen der nationale Dichter gab. ${ }^{22}$

Schließlich besaß Scott laut Fontane genau die Eigenschaften, welche der spätere Romancier der preußischen Vergangenheit und Gegenwart anstreben sollte: alles in Scotts Romanen entzücke ,durch Liebe und Kenntnis der Heimat, durch Reinheit des Empfindens und der Gesinnung, durch rührende Simplizität und vor allem durch einen sich immer gleich bleibenden Humor.“23 So wurde Scott zum

nach Pesne, Watteau und Chodowiecki gestellt wurden, erscheinen läßt, kann uns das Fremdartige vergessen machen" (S. 58-59).

${ }^{19}$ Fontane, Dichtern (Fußnote 1), S. 159-160 (Aufzeichnung nach 1874).

${ }^{20}$ Ebd., S. 160.

${ }^{21}$ Ebd., S. 53 (Scott-Hommage zum 100. Geburtstag, 1871).

22 Ebd., S. 71.

${ }^{23}$ Ebd., S. 62. 
„Shakespeare der Erzählung.“24 Und Shakespeare ist es natürlich auch, der im Drama das National-Historische mit einer universellen Ausstrahlung zu verbinden weiß. Zwar habe man dies auf deutschen Bühnen oft nicht erkennen können, weil „man bei uns den Shakespeare nur für die Gebildeten spielt“; man vergleiche aber „eine deutsche Aufführung des Shakespeare mit einer englischen“, und man würde begreifen, wie ununterbrochen „die Traditionen der englischen Shakespeare-Bühnen“ bis „zu Shakespeare selbst hinauf“ reichen. ${ }^{25}$ Würde man „in Deutschland den unerschütterlichen Glauben“ nicht länger hegen, Shakespeare am besten zu verstehen und zu spielen, wäre die Wirkung der „eigentlich historischen Szenen“ der Königsdramen und insbesondere von deren „historisch-romantische[n] Feingehalt" eine gänzlich andere. ${ }^{26}$

Damit erscheinen Fontanes Vorstellungen zu diesem Themenkomplex - der Treue des Dichters zur Geschichte, vor allem zur Geschichte des eigenen Landes - relativ fassbar und konsequent, trotz aller evidenten Anfechtbarkeit. Man versteht in etwa, was Fontane verlangt und was er ablehnt. Gäbe es nicht hierzu eine Schwenkung, die sich so unvermittelt einstellt, dass man zunächst auf einen besonders eklatanten Fall Fontane'schen Widerspruchsgeistes schließt, der sich ja am ehesten gegen die eigenen Meinungen regt. ${ }^{27}$ Kleists Homburg-Drama, dem er 1872 wegen dessen Missachtung beinahe jeglicher historischen Wirklichkeit die künstlerische Berechtigung so vehement absprach, betrachtet er in der Rezension einer Aufführung von 1876 aus entschieden anderer Sicht. Nun ist es Kleists „schönstes und vollendetes Stück“, überhaupt ein vollendetes Kunstwerk, unübertreffbar in der Wahl des Vorhabens wie auch in dessen Verwirklichung. Alles, was Fontane vier Jahre vorher so verstimmte, wird jetzt zum unentbehrlichen Bestandteil eines überwältigenden Kunsterlebnisses, das nur durch die siegreiche Überwindung des zuvor Befremdenden und Empörenden ermöglicht wird. Der Verrat am Stoff war nur Taktik, und das Stück bringt alles ins Lot:

Es äußert eine von Akt zu Akt sich steigernde, rückwirkende Kraft, die so groß, so erobernd ist, daß wir des letzten Restes von Mißmutes, den die scheinbar romantisch-willkürliche Exposition in uns geweckt hatte, nicht nur quitt werden, sondern uns auch schließlich zu dem halb widerwillig, halb freudig gegebenen Geständnis bequemen: wenn wir das Stück in seiner Schönheit und Macht überhaupt wollen, so müssen wir auch das wollen, was uns an ihm verdroß. Ein Triumph der Kunst, der sich in allen Kleistschen Arbeiten ausspricht, in diesem Prinzen von Homburg aber vielleicht am meisten. Die Klarheit und Konsequenz des Gewollten, das uns überkommene Gefühl absoluter künstlerischer Notwendigkeit, entwaffnen zuletzt jeden Widerspruch und zwingen uns, auch das uns Widerstrebende - das doch seinerseits erst das

${ }^{24}$ Ebd., S, 61.

${ }^{25}$ Ebd., S. 33-36 (Tagebuch, London 1856 sowie Einleitung zum Londoner Theater, 1860).

${ }^{26}$ Fontane, Theaterkritiken (Fußnote 15), S. 43-35 (Besprechung von Heinrich IV. 1. Teil, $1873)$.

27 Vgl. Thomas Mann in seinem Aufsatz (1910) „Der alte Fontane“: „Ein unsicherer Kantonist. Hat er nicht als Theaterkritiker einmal gestanden, eigentlich könne er immer gerade so gut das Gegenteil sagen?", in Thomas Mann, Gesammelte Werke, Band 9, Frankfurt am Main 1974, S. 30. 
eine Vollkommenheit darstellende Ganze wieder zu dem macht, was es ist - an den Anerkenntnissen dieser Vollkommenheit teilnehmen zu lassen. ${ }^{28}$

Angesichts der Intensität der jeweiligen Urteile (jubelndes Insistieren hier, und vier Jahre davor ein fast rabiater Wiederholungsdrang in der Ablehnung) scheint hinter dem Gesinnungswandel mehr zu stecken als die bekannte Fontane'sche Vorliebe für das ,,sowohl als auch“. Aus dem selben Grund scheitert der Versuch, die beiden Haltungen Fontanes zu koordinieren, wie in Peter Goldhammers Anmerkungen zur Rezeptionsgeschichte des Dramas. Fontanes ablehnende Aufzeichnungen, die er nie veröffentlichte, „dienten nur der Selbstverständigung“, in der Rezension von 1876 ,versuchte er sein Urteil zu objektivieren." ${ }^{29}$ Eine solche Harmonisierung der Äußerungen Fontanes kann aber nicht gelingen: zu unterschiedlich, und auch zu konsequent erscheinen sie in ihrer jeweiligen Logik. Eine ganz andere Erklärung, die sich von Fontanes Biographie ableitet, bietet Günther de Bruyn:

Zwischen der Lektüre-Aufzeichnung von 1872 und der späten „Homburg“-Kritik in der Vossischen Zeitung liegt die vielleicht entscheidendste Episode seines Lebens: sein Bürokraten-Erlebnis, seine Anstellung im Staatsdienst, die er erschreckt und verstört schnell wieder aufgibt, durch die er aber klarsichtig wird. In der preußischen Amtsstube, die von ihm nicht nur Gehorsam, sondern auch geistige Selbstverleugnung verlangt, wird sozusagen der Romanschreiber und Theaterkritiker geboren. Die „Homburg“-Rezension, die er nun schreibt, ist ein Indiz für die neugewonnene Realitäts- und Kunstoffenheit. Seine Bedenken sind noch da, werden aber durch Kleists Größe besiegt. ${ }^{30}$

De Bruyns Darstellung verdient schon deswegen Aufmerksamkeit, weil er ein besonders gut placierter Kenner der Materie ist (und auch weil seine Meinung von dem ausgezeichneten Fontane-Interpreten Helmuth Nürnberger übernommen wird $\left.^{31}\right)$. Überzeugen kann sie trotzdem nicht. Eine Verbindung zwischen Fontanes desillusionierender Erfahrung mit der geisteserstickenden Atmosphäre ,preußischer Amtsstuben" einerseits, und andererseits mit den weitreichenden Ambivalenzen der späteren Gesellschaftsromane, besteht zwar mit Sicherheit. Eine derartige Verbindung zu Fontanes ästhetischen Urteilen ist aber kaum auszumachen; diese sind vor 1876 keineswegs unfreier oder gehemmter als später, selbst wenn sie nach wie vor allerhand Widersprüche aufweisen dürfen. Laut de Bruyn findet zwischen den beiden Homburg-Urteilen eine politisch bedingte Blickerweiterung in Kunstdingen bei Fontane statt: „Fontanes politisch eingeengter Blick macht ihn blind für die Kunst. Das wird ihm erst sichtbar, wenn sein Blick klar, also kritisch wird.“32 Doch um die Freiheit und Individualität seines „Blickes“ und die Unverblümtheit seines Ausdrucks schon vor 1876 zu belegen, genügt die Lektüre

${ }^{28}$ Fontane, Theaterkritiken (Fußnote 15), S. 79-80.

${ }^{29}$ Kleist, Dramen, Band 2, Frankfurt am Main 1986, S. 718.

${ }^{30}$ Günther de Bruyn, „Preußen, deine Dichter“ in Lesefreuden: über Bücher und Menschen, Frankfurt am Main 1995, S. 19-20.

${ }^{31}$ Helmuth Nürnberger, Fontanes Welt, München 2007, S. 501.

32 De Bruyn, op. cit., S. 19. 
seiner Anmerkungen zu Goethe von Juli 1870, als ihn die allseits herrschende „patriotische Erregung“" sehr enervierte: „In diesen Nöten fliehe ich zum alten Goethe und lese die ,Natürliche Tochter" und die ,Wahlverwandtschaften'. Ich bewundere es und finde es tief-langweilig. Als Beobachtung des Lebens und Weisheits-Ansammlung klassisch, aber kalt und farblos. “33

Zudem: wenn de Bruyn in der Homburg-Rezension von 1876 einen Befreiungsschlag erkennen will, erhebt sich die Frage, wie man mit all dem reaktionären und grimmig-dumpfen Spott über den „Bankrott der Afterweisheit des vorigen Jahrhunderts" ins Reine kommen mag, der sich beispielsweise 1883 in einem Brief an Fontanes Frau äußert: „Das Unheil, das Lessing mit seiner Geschichte von den drei Ringen angerichtet hat, um nur einen Punkt herauszugreifen, ist kolossal. Das „Seid umschlungen Millionen“ ist ein Unsinn. Hoheitsaufgaben, die doch nicht gelöst werden können, verwirren die Menschheit nur. ${ }^{34}$

Der Wandel in Fontanes Haltung zum vaterländischen Drama seines großen preußischen Vorgängers erklärt sich also nicht durch einen vermeintlich freieren und kritischeren Blick auf preußische Verhältnisse. Eine andere Erklärung deutet sich am Ende der jubelnden Rezension an, nachdem Fontane die schauspielerischen Leistungen des Abends und insbesondere diejenige des Darstellers des Kurfürsten besprochen hat. „Herr Berndal gab den großen Kurfürsten. Diese Rolle, wenn sie überhaupt noch in die vorerwähnte Kategorie gehört, bildet doch jedenfalls ihre Spitze und Verklärung.“ 35 Auf den Begriff der „Verklärung“ - hier wohl im Sinne von „Apotheose“ - kommen wir kurz zurück. Davor aber lesen wir, wie Fontane zum Schluss die Wirkung der Aufführung auf ihn zusammenfasst:

Die Last des Tages - oft auch ganz speziell die Last des Abends - fällt von einem ab, und die reine Luft wirklicher Kunst erquickt den Ermüdeten und facht die alten Hoffnungen auf goldene schöne Tage wieder an. Und rücken diese Tage auch immer wieder hinaus wie die Bilder der Fata Morgana, es ist doch ein Glück, sie dann und wann als bloße Verheißung erscheinen zu sehen. ${ }^{36}$

${ }^{33}$ Fontane, Dichtern (Fußnote 1), S. 77 (Brief an Karl Zöllner vom 23. Juli 1870). Am 29. April 1890 an Georg Friedländer Ähnliches: „Das Überlieferte ist vollkommen schal und abgestanden; wer mir sagt, ,ich war gestern in Iphigenie, welch Hochgenuß!' der lügt oder ist ein Schaf und Nachplapperer" (ebd., S. 80). Der Ausdruck ist gegenüber 1870 etwas schärfer, aber der Gedanke war bereits damals vorhanden.

${ }^{34}$ Ebd., S. 77-78. Die späten Briefe an Georg Friedländer sind bekanntlich auch keineswegs frei von solchen Brümmigkeiten. Was Lessing angeht, mag zu Fontanes Entlastung die Tatsache dienen, dass er im 19. Kapitel von Effi Briest seine Kritik der Ring-Parabel in den Mund des alles andere als geistreichen Güldenklee legt.

35 Fontane spricht eine Seite davor von der „Mischgattung von Grandseigneur und alter Kriegsgurgel“, welche Berndal ,in allen Graden und Erscheinungsformen“ darzustellen verstehe. Da der bisher zitierte Band Theaterkritiken (Fußnote 15) hier - wie manchmal, wenn weniger vom Stück als von den Schauspielern die Rede ist, - eine (wie stets, gekennzeichnete) Kürzung vornimmt, wird jetzt aus dem Band 22,1 von Fontane, Werke, München 1964, S. 510-511 zitiert („Causerien über Theater, 1. Teil").

${ }^{36}$ Ebd., S. 511. 
Wie manchmal in Fontanes Theaterrezensionen ist hier die Grenze zwischen dem, was er über das Stück, und dem, was er über die Aufführung sagt, nicht ganz ersichtlich. Vermutlich meint er in den soeben zitierten Zeilen beides. Sicher ist jedenfalls, dass er Kleists Homburg in dem erhabenen aber auch nicht leicht erfassbaren Reich der Utopie ansiedelt. Utopie, oder Nirgendwo; eine Fata Morgana, die erhebt und beflügelt, selbst wenn ihre Existenz fraglich ist; eine beglückende „Verheißung“. Dieses Wort - wie natürlich auch dasjenige von „Verklärung“, das eine so kapitale Bedeutung für Fontanes ästhetische Positionen besitzt ${ }^{37}$ - entstammt dem Bereich der Theologie und bewegt sich oft in Sphären der Mystik, die auf den ersten Blick Fontanes Realismus-Begriff fern liegen. Zumal es sich hier um eine „bloße VerheiBung" handelt, also um eine letzten Endes unwirkliche. Dennoch braucht man sie wie die Luft zum Atmen, oder genauer gesagt wie die „,reine Luft wirklicher Kunst“, welche die preußische Wirklichkeit hier durchzieht. Fontane lobt oder lobpreist jetzt den Prinzen von Homburg, weil er ihn nunmehr so versteht, wie Kleist ihn verstanden hat: als preußische Utopie, als Mythos, als Gründungsmythos des Staates Preußen.

Natürlich hatte Fontane recht, als er 1872 die Punkte auflistete, die Kleists Stück von der eigentlichen vaterländischen Geschichte entfernten. Hiervon nun einige:

[...] in Fehrbellin gibt es kein Schloss, ein gefangengesetzter Prinz kann nicht auf eine halbe Stunde eine Prinzessin besuchen, brandenburgische Obersten versammeln sich nicht, in harmloser Meuterei, zu einer Sturmpetition, kein brandenburgisch Regiment (wie die Pappenheimer, die ihren Oberst fordern) rückt ohne Befehl vor das kurfürstliche Schloss, kein Kottwitz hält eine Rede über das Tötende der Disziplin und über das gelegentliche Recht der „freien Aktion“ $[\ldots]^{38}$

Dennoch verkörpert der Kleist'sche Kottwitz wie niemand sonst das wahre Preußen. Fontanes Sinn für Paradoxe hat ihn später, im Graf Petöfy, dazu geführt, sein überzeugendstes Bekenntnis zum Preußentum in den Mund eines erklärten Gegners sowohl Preußens als auch des Protestantismus zu legen. Der liguorianer Pater Feßler erläutert den Grund für Preußens Vorherrschaft gegenüber Österreich. Die Preußen seien innerlich frei:

Sie prüfen die Dinge, sind kritisch und leben selbstständig aus sich heraus. Und das ist ein Heilsweg [...] unter richtiger Voraussetzung der einzige Weg, der zum Heile führt. [...] Es gibt eine höchste Lebensform und diese höchste Lebensform heißt: „,in Freiheit zu dienen.“ Das Dienen aus bloßem Zwang heraus ist todt und erst aus einem selbstgewollten, weil als unerläßlich erkannten Verzicht auf die Freiheit erblüht uns der echte, welterlösende Glauben. ${ }^{39}$

So ist Kottwitz - „Frei und für mich, im Stillen, unabhängig“40 - der treuste Diener seines Herrn. Dieser hätte in Wahrheit - erneut hat der indignierte Fon-

${ }^{37}$ Zur „Verklärung“ als überaus polyvalenter Kategorie in Fontanes kritischem Vokabular vgl. den Abschnitt zu diesem Begriff im Kapitel „Fontanes Poetik“ von Hugo Aust in: Fontane-Handbuch, hg. v. Christian Grawe u. Helmuth Nürnberger, Stuttgart 2000, S. 427-429.

38 Fontane, Dichtern (Fußnote 1), S. 95.

39 Theodor Fontane, Graf Petöfy, Berlin 1999, S. 18 (3. Kapitel).

${ }^{40}$ Heinrich v. Kleist, Prinz Friedrich von Homburg, Stuttgart 2011, S. 84 (5. Akt, 5. Auftritt, Vers 1592). 
tane von 1872 völlig recht - auch nie gesagt, der vom Kriegsgericht verurteilte Prinz ,mag selber entscheiden; glaubt er, daß ihm Unrecht geschehen sei, so ist er frei." "41 Anno 1876 weiß Fontane aber, dass die eklatant unhistorische Haltung des Kurfürsten Friedrich Wilhelm bei Kleist die voraussehbare Bestätigung des preuBischen Credo durch den Prinzen bewirkt: ,in Freiheit zu dienen“. Eine historische Unwahrheit wird damit zur vaterländischen Parabel, von Kleist in Szene gesetzt.

Fontane erkennt damit bei Kleist einen Vorgang, dem er in den Jahren davor, während der Niederschrift der drei Kriegsbücher, oft begegnete und auch in der Praxis huldigte. Preußens Siege brauchten Helden, brauchten anschauliche, sich einprägende Beispiele von Opfermut und Heldentod. Die glorreichen Siege brauchten glorreiche Taten, die sich in zugespitzter Gestalt zu Symbolen erheben konnten. Im Deutsch-Dänischen Krieg 1864 steht die Großtat des einfachen Pioniers mit dem volkstümlichen Namen „Klinke“ beispielhaft dafür - und auch für die Unsicherheit darüber, ob die gefeierten und immer wieder tradierten Taten in solcher Heldenhaftigkeit wirklich stattgefunden haben: „Welche Lesart auch immer die richtige sein mag, das Volk wird sich seinen ,Klinke‘ ebenso wenig nehmen lassen wie seinen ,Froben“; mit der historischen Aufhellung - die ohnehin höchst mißlich ist und oft noch mehr vorbeischießt als die Dichtung - ist dem Bedürfnis des Volks nicht immer am meisten gedient. “42

Wichtig für Fontanes Homburg-Beobachtungen ist natürlich die Anspielung auf Froben. Nicht nur deswegen, weil seine Opfertat in Homburg ausführlich geschildert wird (2. Akt, 8. Auftritt), sondern weil er als Adliger auch für Preußens Aristokratie als Vorbild dient - er wird im Drama ja von dem Prinzen bewundert und von der Kurfürstin und Natalie beweint, und noch in Effi Briest von Effi selbst als Modell des wahren, des Herzensadels gepriesen (Schluss des 8. Kapitels, im ersten Gespräch mit Gieshübler). Frobens vermeintliche Heldentat ist ohne historische Grundlage. Fontane ist zwar, fast möchte man sagen von der Veranlagung her, Skeptiker; aber er lässt Vater und Tochter Briest (und den Apotheker Gieshübler wohl auch dazu) an Frobens Tat glauben, die ja für sie zur Grundlage ihres Preußentums gehört. Fontane weiß, bei Bedarf seine Skepsis zu dämpfen: „In den Kriegsbüchern kommt diese Skepsis selten direkt zum Vorschein; sie wird unterdrückt, und stattdessen spricht der ,Balladendichter’، “43 Auch die Ballade ist Zuspitzung, Kondensation, Erhöhung durch Entfernung von Beiwerk, Ambiguitäten und Nuancen. Gerade in der Wiedergabe von Kriegstaten - wofür das sprechendste Beispiel dem 3., dem Berliner Teil von Fontanes vaterländischem

${ }^{41}$ Fontane, Dichtern (Fußnote 1), S. 95.

42 Theodor Fontane, Der Schleswig-Holsteinische Krieg, hier zitiert nach dem Kapitel von John Osborne „Die Kriegsbücher“ (Abschnitt „Mythisierung und Entmythisierung“) in Fontane-Handbuch (Fußnote 37), S. 861.

43 Ebd., S. 861. Das Wort „Balladendichter“ übernimmt Osborne einer Studie von G. Friedrich. Osbornes Buch Theodor Fontane: Vor den Romanen. Krieg und Kunst, Göttingen 1999, gehört zu den wichtigsten Fontane-Veröffentlichungen der letzten 20 Jahre. 
Vor dem Sturm entstammt. Beim Treffen des literarischen Vereins „Kastalia“ wird zuerst Lyrik, dann Prosa vorgestellt. Der Gast Hansen-Grell trägt seine Ballade über den preußischen Kavalleriegeneral Seydlitz (1721-1773) vor. In der Diskussion danach wird zunächst bemängelt, dass die gewiss sehr lebhaften Verse wegen der extremen Knappheit der Darstellung nur von ausgesprochenen Kennern der preußischen Geschichte verstanden werden können. Dem widerspricht aber ein anderer Gast, der polnische Graf Bninski. Die Details seien zwar knapp und wenig, ,aber dies wenige ist genug, weil es ein Ausreichendes ist.“ Seine Erfahrungen mit Volksliedern hätten ihm gelehrt, „daß das sprungweise Vorgehen zu den Kennzeichen und Schönheiten dieser Dichtungsgattung gehört. Die Phantasie muß nur den richtigen Anstoß empfangen; ist dies geglückt, so darf man kühn behaupten: ,je weniger gesagt wird, desto besser' ““44

Danach wird ein Prosa-Stück vorgelesen: ein im Ton diskret gehaltener, mit zahlreichen wohlgesetzten Details versehener, in seiner Humanität bewegender Bericht über Kriegshandlungen nur zwei Jahre davor mit der Überschrift „Erinnerungen aus dem Krieg in Spanien“. Der Bruder von Eugen von Hirschfeld erzählt darin vom „Gefecht bei Plaa“, in dem dieser tödlich verwundet wurde. Überall Nuancen; alles Heldentum liegt in der menschlichen Würde und Zurückhaltung im Angesicht des Todes. Weshalb am Ende der Lektüre der Graf Bninski - der in der selben Schlacht auf der anderen, der Napoleonischen Seite kämpfte, wieder das Wort ergreifen kann, das er an den Erzähler, den Bruder des Gefallenen, richtet: „Was Freund, was Feind! An gleicher Stelle die gleiche Gefahr. Ich bitte Sie darauf hin als einen mir theuer gewordenen Kameraden begrüßen zu dürfen.“45

Erzählende Prosa, wie später meist bei Fontane, relativiert, durch ihre Fülle an Details; erzählende Lyrik, wie hier die Ballade (die ja in Wirklichkeit nicht von Hansen-Grell, sondern von Fontane selbst geschrieben wurde), amplifiziert, wegen der Verknappung aller Details. Das Homburg-Drama, nicht nur und nicht einmal in erster Linie, weil es ein Vers-Drama ist, liegt der Ballade näher als dem Bericht. Fontanes vaterländischer Roman unterhält in seinem oft überwuchernden Detailreichtum dagegen nur wenige Verbindungen zur Ballade, trotz aller Märchenelemente um die „Märchenprinzessin“ Marie und die gute Hexe Hoppenmarieken. Die Verbindung zu Kleist ist zuerst eine andere, historische. Fontane schreibt einen Roman über den Aufstand gegen Napoleon, den Kleist mit seinem Homburg-Schauspiel herbeizurufen hoffte. In beiden Werken geht es ja um den Gehorsam, den man in Preußen dem Kurfürsten bzw. dem König noch schuldet, wenn Ungehorsam dem Lande dient. ${ }^{46}$

${ }^{44}$ Fontane, Vor dem Sturm, Band III/IV (Fußnote 12), S. 107-108.

45 Ebd., S. 118.

46 In einem Zeitungsartikel über Vor dem Sturm aus dem Frühjahr 1981 wies Joachim Kaiser auf die unverhoffte Aktualität dieses historischen Romans hin: „Der Machthaber, der lieber ein Bündnis mit dem Feinde schließt als mit dem eigenen, aus dem Geleise des Gehorsams gekommenen Volke, ein solcher Machthaber erinnert beängstigend an den polnischen Ministerpräsidenten des April 1981 - der sich vielleicht doch lieber auf Rußland als auf die schwer kalkulierbare Gewerk- 
Doch nicht darin liegt die wichtigste Beziehung des Romans zum Homburg. Fontanes Erstling schildert eine bedeutungsvolle und folgenreiche Etappe der vaterländischen Geschichte: wie Preußen, einstmals groß und später schwach - durch eigene Schuld am Boden liegend und dem Feinde ausgeliefert - sich dann wieder erhob, aus eigener Kraft und unter Entfernung alles Fremden aus Ost und West. Vor dem Sturm konsolidiert, kodifiziert literarisch diese Etappe. Ambivalenzen sind nicht ausgeschlossen, aber der Roman glorifiziert nicht nur einen Sieg, sondern die Wiederauferstehung eines Staates, der Bestand haben sollte bis zur Vollendung seines Auftrages in der Schlacht bei Sedan und der Proklamation des Reiches in Versailles. Die erste Erweiterung und Konsolidierung Preußens unter Friedrich II. war schon längst ausreichend geschildert und besungen worden; in Vor dem Sturm begnügt sich denn auch Fontane mit der freilich sehr effektvollen Übernahme älterer Memoiren-Literatur über den alten König. ${ }^{47}$ Auch der Tag, der allem Anschein nach kapitale Spuren in den Charakter des Kronprinzen Friedrich hinterließ - der „Tag von Küstrin“, der Tag der Enthauptung seines Freundes Katte, der 6. November 1730 - lag in ausführlicher Schilderung bereits vor; Fontane lieferte hierzu seinen Beitrag 1879 mit einem 40-seitigen Aufsatz: „Die Katte-Tragödie“. Zum Beginn setzt Fontane diesen „Tag von Küstrin“ in direktem Zusammenhang mit dem „Tage von Fehrbellin“, dem 18. Juni 1675; er möge als dessen „Gegenstück“ gelten. Preußens Gegenwart entstammt den beiden Daten: „Mit diesen beiden Tagen, dem heiteren 18. Juni und dem finsteren 6. November, beginnt unsere Großgeschichte. Aber der 6. November ist der größere Tag, denn er veranschaulicht in erschütternder Weise jene moralische Kraft, aus der dieses Land, dieses gleich sehr zu hassende und zu liebende Preußen, erwuchs. “48

Mag „Küstrin“ der größere Tag gewesen sein - „Fehrbellin“ war dagegen der erste. Dann und dort fing alles an. Doch dazu gab es innerhalb aller schriftlichen Gestaltungen dieses Tages nichts, was Verbindliches, Endgültig-Gültiges zu diesem Gründungsmoment alles Preußischen bieten konnte. Es sei denn, man nehme Kleist, und nehme damit sämtliche historischen Unwahrheiten in Kauf, um Preußens singulären Anspruch „in Freiheit zu dienen“ dichterisch zu demonstrieren. ${ }^{49}$

schaft „Solidarität“ einläßt. Dazu Fontane, wie ein kluger Leitartikler von heute: ,Denn er ist ganz auf Ordnung gestellt. Mit einem einheitlichen Feinde weiß er, woran er ist, mit einer vielköpfigen Volksmasse nie'.“ „Idealisierung und Zeitkritik. Theodor Fontanes erster Roman Vor dem Sturm“, nachgedruckt in Joachim Kaiser, Was mir wichtig ist, Stuttgart 1996, S. 141-154; hier S. 154.

${ }^{47}$ Fontane, Vor dem Sturm, Band I/II (Fußnote 12), S. 216-217 (2. Band, 7. Kapitel).

48 Theodor Fontane, Wanderungen durch die Mark Brandenburg. Das Oderland, Berlin 1994, S. 299.

${ }^{49}$ Angesichts dieser Bedeutung von Kleists Drama für Fontanes Preußen-Verständnis wäre folgender Satz in Hugo Austs Artikel zum Thema zu modifizieren: „An einem marginalen Moment, Fontanes Homburg-Rezeption, zeichnen sich Partikel einer Poetik der realistischen Schaffensmethode ab“; marginal ist der Moment nur scheinbar. Der Aufsatz ist aber sehr lesenswert. „Der Prinz von Homburg - ein ,Waschlappen'? Fontanes Irrungen und Wirrungen mit Kleist", in Hugo Aust et al., Hg., Fontane, Kleist und Hölderlin, Würzburg 2005, S. 137-146, hier S. 144. Darin auch zu empfehlen: 
Als Fontanes Homburg-Rezension 1876 erschien, war Preußens Werdegang seit Fehrbellin seit fünf Jahren vollzogen - und dichterisch in allen wesentlichen Etappen zwischen 1675 und 1815 nachgezeichnet. Fontane, der 1883 in Schach von Wuthenow die Vorgeschichte zu Vor dem Sturm nachlieferte, sparte die Zeit vor der Reichsgründung aus seinen Gesellschaftsromanen weitgehend aus; die preußische Gegenwart erschien ihm eindeutig vordringlicher. Die Aporien, die er in ihr fand - in seinem ,gleich sehr zu hassenden und zu liebenden Preußen“ - waren bereits von Kleist vorweggenommen. Am deutlichsten wohl in zwei kontrastierenden, und sich dadurch ergänzenden Bühnenhandlungen über die Freiheit des (oder der) einzelnen und dem Gebot des Dienens, das vom Kollektiv ausgeht. In Penthesilea, wo Preußen sich als der mythische Staat der Amazonen verkleidet, scheitert der Ausgleich zwischen Freiheit und Dienen, und die Hauptfigur wird seelisch zermalmt (und ihr Geliebter dafür noch physisch). In der Homburg-Utopie gelingt grandios, mit Kanonenschüssen, die Versöhnung der Gegensätze. Ein vergleichbares Paar bildet sich aus Kleists Erzählungen heraus. Preußen und dessen Geist wird nach Italien verlegt, und die Fata Morgana der Vereinbarkeit von Gefühl und Gesetz wird, nach langem Kampf, wahr (Die Marquise von O...); auch nach Chile wird Preußisches versandt, aller katholischen Szenerie zum Trotz, wo sich aber die Hoffnung auf eine neue, dem Menschen gemäße Gesetzlichkeit in Schrecken zerschlägt (Das Erdbeben in Chili). Wie Kleist schwankt auch Fontane im Hinblick auf Preußens Fähigkeit, sich im Sinne des Großen Kurfürsten auf Kleists Bühne das Gesetz dadurch zu festigen, dass er den freien Willen zu dessen Sockel macht. Doch anstatt eines Sockels entdeckt Fontane in den späteren, immer noch „vaterländischen“ (da das Vaterland betreffenden) aber nicht mehr unbedingt patriotischen Romanen vor allem „Hülfskonstruktionen“. Mit diesem Begriff umschreibt Wüllersdorf (gegen Ende von Effi Briest) im Gespräch mit Instetten die Ablenkungen und Selbsttäuschungen, die es in Preußen einem ermöglichen, die eigene Resignation oder Verzweiflung im Schach zu halten: „einer, dem auch viel verquer gegangen war, sagte mir 'mal: ,Glauben Sie mir, Wüllersdorf, es geht überhaupt nicht ohne ,Hülfskonstruktionen'.' Der das sagte, war ein Baumeister und mußt' es also wissen. Und er hatte recht mit seinem Satz. Es vergeht kein Tag, der mich nicht an die ,Hülfskonstruktionen' gemahnte. ${ }^{50}$

Kennzeichen des Fontaneschen Spätwerks ist der Versuch, Preußens durch Sedan und Versailles verbriefte Glorie an der preußischen Wirklichkeit zu ermessen. Da die Ergebnisse trostlos ausfallen, greift man nach den Lösungen, die sich eben bieten: des alten Briest Ehrfurcht vor dem „weiten Feld“, des alten Stechlin Zuversicht an das Fortleben des Sees und seiner Legende auch nach dem Niedergang der Stechline. Für den 1876 noch nicht alten, aber schon reifen Fontane, der kurz davor steht, Preußens erster Romancier zu werden, bestand wohl die Lösung

Bettina Pleit, „Preußische Ideen. Preußenbild und ,Vaterlandsliebe’ bei Kleist und Fontane“, S. 121135 sowie Barbara Dölemeyer, „Prinz Friedrich von Homburg - Historie und Dichtung“, S. 11-29.

50 Theodor Fontane, Effi Briest, Berlin 1998, S. 342 (36. Kapitel). 
in der Verheißung und Verklärung Preußens, die vor ihm Kleist erträumte. Sogar eine Apotheose kann zuletzt eine „Hülfskonstruktion“ sein.

\section{Literatur}

\section{Primärliteratur}

Fontane, Theodor: Werke, Band 22,1. München 1964.

Fontane, Theodor: Wanderungen durch das Mark Brandenburg. Das Oderland. Berlin 1994.

Fontane, Theodor: Effi Briest. Berlin 1998.

Fontane, Theodor: Die Saison hat glänzend begonnen. Theaterkritiken. Berlin 1998.

Fontane, Theodor: Ach, es ist schlimm mit den Dichtern. Über Literatur, Autoren und das Publikum. Berlin 1999.

Fontane, Theodor: Graf Petöfy. Berlin 1999.

Fontane, Theodor: Vor dem Sturm. Berlin 2011.

Kleist, Heinrich von: Briefe. Frankfurt am Main 1986.

Kleist, Heinrich von: Prinz Friedrich von Homburg. Stuttgart 2011.

Mann, Thomas: Gesammelte Werke, Band 9. Frankfurt am Main 1974.

\section{Sekundärliteratur}

Aust, Hugo et al.: Fontane, Kleist und Hölderlin. Würzburg 2005.

de Bruyn, Günther: Lesefreuden: über Bücher und Menschen. Frankfurt am Main 1995.

Grawe, Christian und Nürnberger, Helmuth: Fontane-Handbuch. Stuttgart 2000.

Kaiser, Joachim: Was mir wichtig ist. Stuttgart 1996.

Nürnberger, Helmuth: Fontanes Welt. München 2007.

Osborne, John: Theodor Fontane: Vor den Romanen. Krieg und Kunst. Göttingen 1999.

\section{Abstracts}

Fontane war sich dessen bewusst, dass einige seiner Meinungen nur provisorisch, also veränderlich waren. Ein so radikaler Umschwung wie bei seinen zwei Beurteilungen von Kleists „Prinz Friedrich von Homburg“ ist aber selbst für seine Verhältnisse ungewöhnlich. Den Grund für seinen Wandel von empörter Ablehnung zur uneingeschränkten Lobpreisung darf man am ehesten in der utopischen Beschaffenheit von Kleists vaterländischem Fantasiestück suchen.

Schlüsselwörter: Preußen, Vaterland, Gründungsmythos, Apotheose 


\section{Patriotic fictions. Fontane and Kleist}

Few writers have changed their minds as often, and about as many things, as Fontane. His most striking volte-face concerned Kleist's glorification of their common Prussian homeland: the drama Prince Frederick of Homburg. Fontane reviled the work in 1872 and unconditionally sang its praises four years later. Why? Perhaps because Kleist's utopian dream made it possible to suspend disbelief in Prussia's promises, and to pretend they were almost true.

Keywords: Prussia, motherland, constitutive myth, apotheosis

Chris Rauseo

Universität Valenciennes

Fllash - Le Mont Houy

59313 Valenciennes Cédex 9

Frankreich

E-Mail: chris.rauseo@univ-valenciennes.fr 